\title{
Qualitative and quantitative differences between bile ducts in chronic hepatitis and in primary biliary cirrhosis
}

\author{
C A Rubio
}

\begin{abstract}
Aim-Lymphocytic infiltration in the portal triads usually conceals the detection-in haematoxylin and eosin (H\&E) stained sections-of bile ducts in two liver diseases: chronic hepatitis and primary biliary cirrhosis. The aim was to assess the number and the characteristics of the bile ducts in those diseases with the aid of an antibody to cytokeratin 7 (CK7)

Methods-Consecutive sections from 99 liver biopsies were stained with $\mathrm{H} \& \mathrm{E}$ and anti-CK7.
\end{abstract}

Results-In H\&E sections the total number of central bile ducts in the triads was 52 in primary biliary cirrhosis $(n=37), 69$ in chronic hepatitis $(n=43)$, and 30 in miscellaneous cases $(n=19)$. Using anti-CK7, the number of central bile ducts was 276 in primary biliary cirrhosis, 348 in chronic hepatitis, and 96 in miscellaneous cases. Central bile ducts with lumen were found in $93.0 \%$ of chronic hepatitis cases and in $89.5 \%$ of the miscellaneous cases, but in only $13.5 \%$ of the primary biliary cirrhosis cases. Peripheral bile ducts in groups of $\geqslant 4 /$ triad were found in all cases of chronic hepatitis $(100 \%)$ and in $75.7 \%$ primary biliary cirrhosis cases, but only in $10.5 \%$ of the miscellaneous cases. In $21.6 \%$ of primary biliary cirrhosis cases, no bile ducts (central and/or peripheral) were present.

Conclusions-Anti-CK7 detects bile ducts in the triads that are concealed by chronic inflammatory cells. Central and peripheral bile ducts in groups of $\geqslant 4$ were significantly more common in primary biliary cirrhosis and chronic hepatitis than in other liver diseases. The lack of lumen in central bile ducts, as well as the absence of central and/or peripheral bile ducts in CK7 stained liver sections, seem to be valuable additional parameters in the differential diagnosis between primary biliary cirrhosis and chronic hepatitis.

(F Clin Pathol 2000;53:765-769)

Keywords: bile ducts; cytokeratin 7 ; primary biliary cirrhosis

Primary biliary cirrhosis is a disease characterised by an autoimmmune destruction of the biliary epithelial cells that line the small intrahepatic bile ducts, whereas chronic hepatitis is characterised by chronic inflammation of the triads, its main target being the liver paren- chyma that surrounds those triads. Chronic hepatitis is the histological expression of diseases with disparate aetiologies, activities, clinical pictures, and prognosis.

The histological appearance of primary biliary cirrhosis and chronic hepatitis in liver biopsies have been amply described. ${ }^{12}$ However, even histopathologists specialised in liver diseases agree that the differential histological diagnosis between these two diseases can be very difficult in at least $15 \%$ of cases when using conventionally stained histological sections. ${ }^{2}$ This is mainly the result of misleading bile duct damage, predominant lymphocytic piecemeal necrosis (inflammatory necrosis of the periportal adjacent liver parenchyma) in cases with clinical features of primary biliary cirrhosis, or difficulty in identifying bile ducts in portal triads showing pronounced lymphocytic infiltration in chronic hepatitis. ${ }^{3}$ The latter pitfall is perhaps the most common source of error in diagnoses based on haematoxylin and eosin (H\&E) stained sections.

Previous studies using antikeratin antibodies in cases with primary biliary cirrhosis were reported as negative. ${ }^{4}$ Recently, we reported preliminary results on the characteristics of bile ducts stained with anticytokeratin 7 (CK7), ${ }^{5}$ using a monoclonal antibody that reacts with the $54 \mathrm{kDa}$ cytokeratin intermediate filament protein of a large number of epithelial cell types, including many ductal and glandular epithelial cells. In the liver, hepatocytes are unreactive, whereas the epithelial cells of the bile ducts are strongly positive.

The aim of our study was to investigate the structural characteristics and distribution of CK7 stained bile ducts in a relatively large number of liver biopsies from patients with primary biliary cirrhosis or chronic hepatitis.

\section{Material and methods}

Formalin fixed and paraffin wax embedded sections from 99 patients with primary biliary cirrhosis $(n=37)$, chronic hepatitis $(n=43)$, and other liver diseases $(n=19)$ were investigated.

CK7 IMMUNOSTAINING PROCEDURE

Paraffin wax sections were subjected to enzymatic digestion with pronase before staining. After digestion, sections were stained with monoclonal mouse antihuman CK7 (Dako A/S, Glostrup, Denmark), dilution 1/600, in the three stage immunoperoxidase technique.

According to their localisation in the triads, bile ducts were divided into those located in the 
central area of the triad (central ducts) and those seen at the periphery of the triads, near the limiting plate (peripheral bile ducts).

The numbers of bile ducts present in two consecutive portal triads were recorded in $\mathrm{H} \& \mathrm{E}$ and in CK7 stained sections in all 99 biopsies. Cases with fibrosis or cirrhosis were not included.

All sections were analysed without knowledge of the patients' data or of the previous histological diagnosis. After the identity of the $\mathrm{H} \& \mathrm{E}$ and CK7 stained slides was concealed they were randomised, and the number of bile ducts was recorded in consecutive slides, to avoid bias. After assessing the number of bile ducts, the identity of the slides was revealed and they were regrouped by patient and by the type of liver disease.

Results were analysed by the $\chi^{2}$ test and by the Mann-Whitney U non-parametric test.

\section{Results}

Liver biopsies from 99 patients having a final clinical diagnosis of primary biliary cirrhosis $(n=37)$, chronic hepatitis $(n=43)$, or miscellaneous liver diseases $(n=19)$ were reviewed.

In patients with miscellaneous diseases, the final diagnosis was based on the results of the liver biopsies: 12 patients had fatty liver as a result of alcohol or associated with methrotrexate treatment, ${ }^{6}{ }^{7}$ three had liver metastasis, one had alcohol associated hepatitis, one had sarcoidosis, one had a normal liver, and one had drug induced intrahepatic cholestasis.

Of the patients with primary biliary cirrhosis, 32 were women and the remaining five were men. The mean age was 55.4 years (range, 40-66). Of the patients with chronic hepatitis, 26 were women and 17 men. Their mean age was 47.4 years (range, 30-67). Of the patients with miscellaneous liver diseases, 12 were men and seven women. The mean age was 53.2 years (range, 31-69).

Figures 1-5 show examples of $\mathrm{H} \& \mathrm{E}$ and CK7 stained sections.

QUANTITATIVE RESULTS

Number of central bile ducts (HEE stained sections)

The total number of central bile ducts found in the 37 biopsies of primary biliary cirrhosis was 52 , in the 43 biopsies of chronic hepatitis 69 , and in the 19 biopsies of miscellaneous diseases 30.

Table 1 shows that the mean number of central bile ducts/triad found in cases of primary biliary cirrhosis was 1.4 (range, $0-4$ ), in cases of chronic hepatitis 1.6 (range, 1-5), and in miscellaneous cases 1.6 (range, 1-5).

\section{Number of central bile ducts (CK7 stained sections)}

The total number of central bile ducts found in the 37 primary biliary cirrhosis biopsies was 276 , in the 43 chronic hepatitis biopsies 348, and in the 19 miscellaneous disease biopsies 96.

Table 1 shows that the mean number of centrally located CK7 stained bile ducts/triads found in cases of primary biliary cirrhosis was

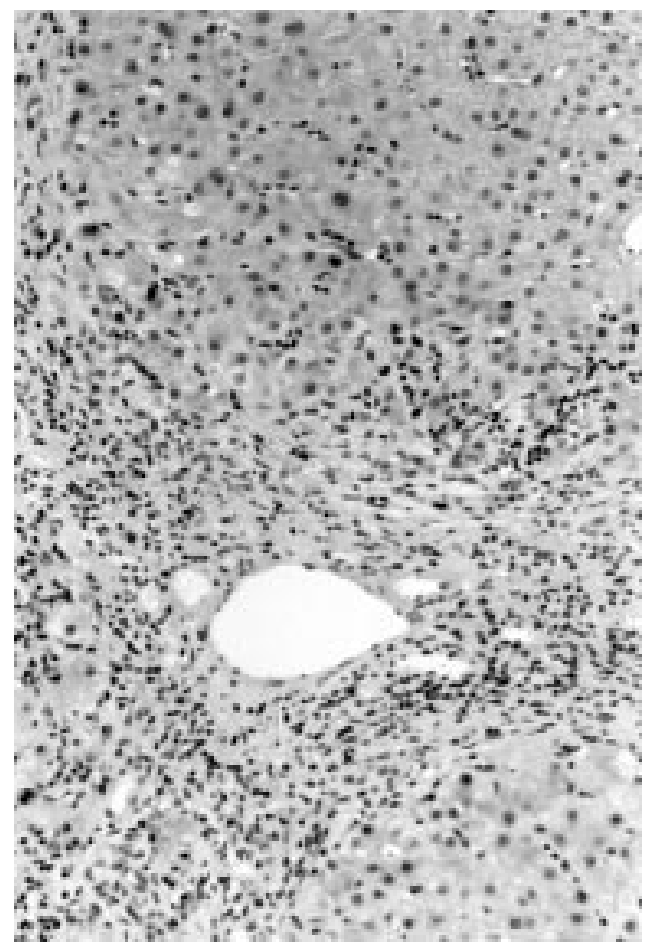

Figure 1 Section stained with haematoxylin and eosin from a liver biopsy in a patient with chronic hepatitis. Bile ducts cannot be clearly identified (magnification, $\times 60$ ).

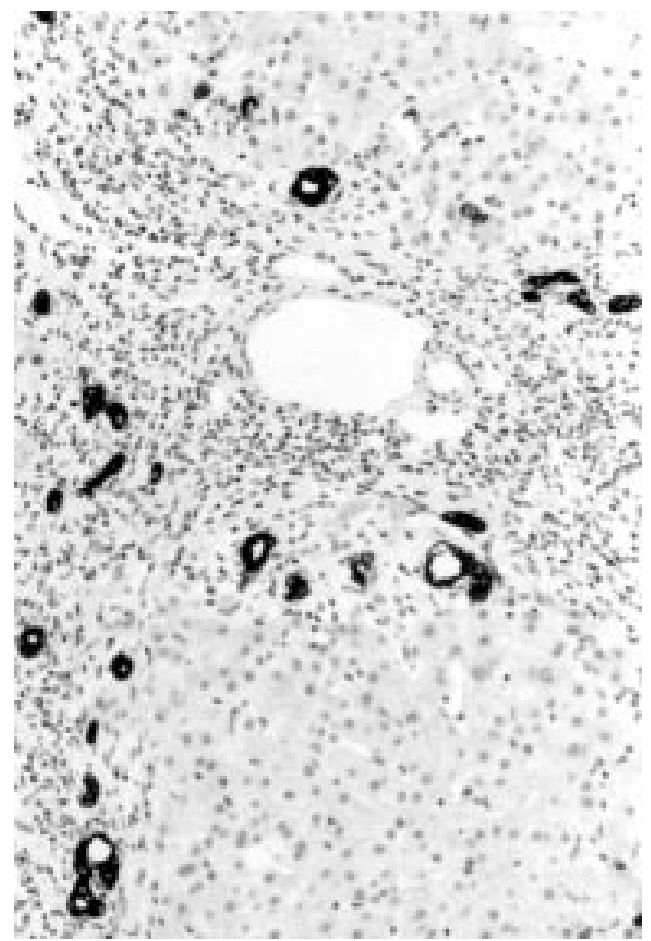

Figure 2 Section from the same liver biopsy as in fig 1 stained with anticytokeratin 7. A moderate number of bile ducts with lumen have been distinctly labelled (magnification, $\times 60$ ).

7.5 (range, 0-40), in cases of chronic hepatitis 8.1 (range, 2-7), and in miscellaneous cases 5.1 (range, 1-6). The difference between primary biliary cirrhosis/chronic hepatitis and miscellaneous liver diseases was significant $(\mathrm{p}<0.001)$.

Table 2 shows that 210 of the $276(76.0 \%)$ central bile ducts recorded in primary biliary 


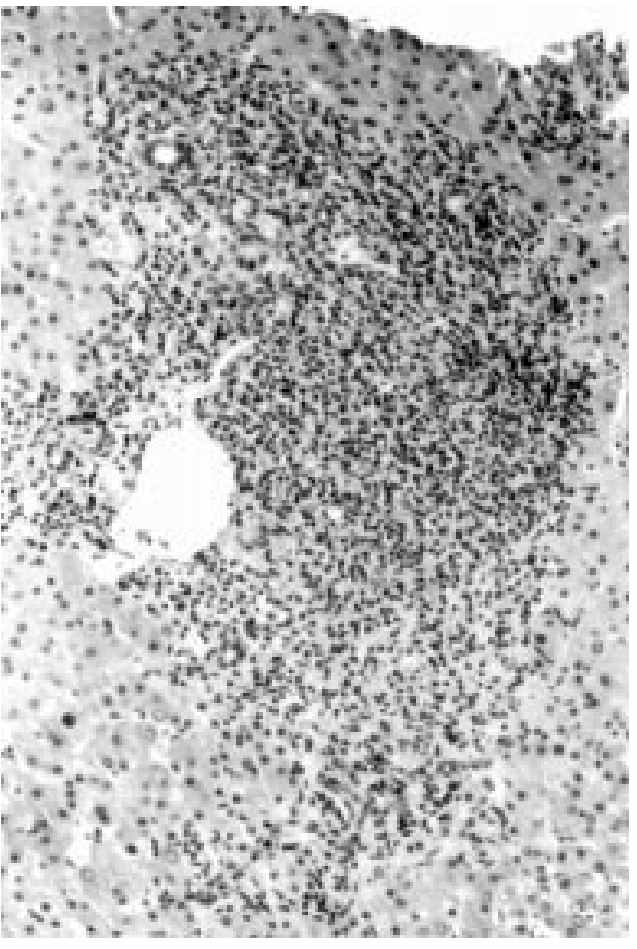

Figure 3 Section stained with haematoxylin and eosin from a liver biopsy in a patient with primary biliary cirrhosis. Only a few bile ducts can be identified (magnification, $\times 60$ ).

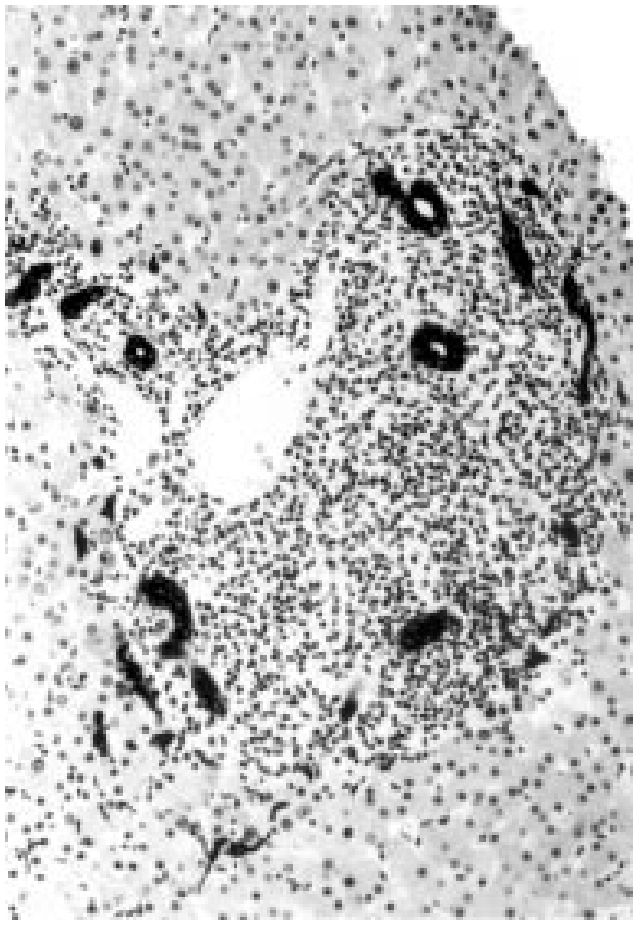

Figure 4 Section from the same liver biopsy as in fig 3 stained with anticytokeratin 7. Central and peripheral bile ducts can now be identified (magnification, $\times 60$ ).

cirrhosis occurred in groups of $\geqslant 4$, that 290 of the $348(83.3 \%)$ central bile ducts present in chronic hepatitis also occurred in groups of $\geqslant 4$, but that only 20 of the $96(20.8 \%)$ central ducts in specimens from patients with miscellaneous diseases occurred in groups of $\geqslant 4$. The difference in the number of central bile ducts between primary biliary cirrhosis/chronic

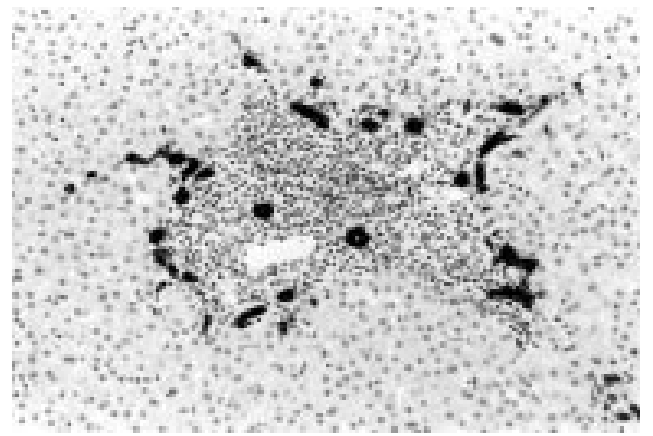

Figure 5 Section from a liver biopsy in a patient with primary biliary cirrhosis stained with anticytokeratin 7. Note the predominance of peripheral ducts (by and adjacent to the limiting plate) without lumen (magnification, $\times 20$ ).

hepatitis and miscellaneous liver diseases was significant $(\mathrm{p}<0.001)$.

Number of peripheral bile ducts (HEE stained sections)

The total number of peripheral bile ducts found in the 37 primary biliary cirrhosis cases was 30 , in the 43 chronic hepatitis cases 28, and in the 19 miscellaneous cases 10 .

Table 1 shows that the mean number of peripheral bile ducts/triads found in $H \& E$ stained sections from patients with primary biliary cirrhosis was 0.8 (range, 0-3), in chronic hepatitis biopsies 0.6 (range, $0-3$ ), and in biopsies from patients with miscellaneous diseases 0.5 (range, 0-3). The number of peripheral ducts was significantly higher ( $p$ $<0.01$ ) in primary biliary cirrhosis/chronic hepatitis than in miscellaneous liver diseases.

Number of peripheral bile ducts (CK7 stained sections)

The total number of peripheral bile ducts found in the 37 primary biliary cirrhosis cases was 332 , in the 43 chronic hepatitis cases 510 , and in the 19 miscellaneous cases 126 .

Table 1 shows that the mean number of peripheral bile ducts/triad detected by CK7 in primary biliary cirrhosis was 9.0 (range, 0-9), in chronic hepatitis 11.9 (range, 4-7), and in miscellaneous cases 6.63 (range, 2-6). The difference in the number of peripheral bile ducts between H\&E and CK7 stained sections was significant $(p<0.001)$ for the three groups investigated.

From table 3 it can be deduced that $\geqslant 4$ bile ducts/triad were found in $89.8 \%$ (298 of 332) of the peripheral bile ducts in primary biliary cirrhosis, in all $(100 \%)$ of the 510 peripheral bile ducts detected by CK7 in chronic hepatitis, but in only $36.5 \%$ (46 of 126 ) of the peripheral bile ducts found in miscellaneous liver diseases stained with anti-CK 7 . The number of liver biopsies with $\geqslant 4$ peripheral bile ducts was significantly higher $(p<0.001)$ in primary biliary cirrhosis/chronic hepatitis than in miscellaneous liver diseases. However, no significant differences were found between primary biliary cirrhosis and chronic hepatitis $(\mathrm{p}<0.6)$.

Of the 37 cases of primary biliary cirrhosis, $28(75.7 \%)$ had $\geqslant 4$ peripheral bile ducts/triad. Whereas all the 43 chronic hepatitis cases 
Table 1 The mean* number of central and peripheral bile ducts found in one portal triad in 99 liver biopsies. Sections stained with haematoxylin-and eosin (HEE) and anticytokeratin 7 (CK7)

\begin{tabular}{|c|c|c|c|}
\hline $\begin{array}{l}\text { Position of bile ducts } \\
\text { in the triads }\end{array}$ & $\begin{array}{l}P B C \\
(n=37)\end{array}$ & $\begin{array}{l}\mathrm{CH} \\
(n=43)\end{array}$ & $\begin{array}{l}\text { Miscellaneous } \\
(n=19)\end{array}$ \\
\hline Central (H\&E) & $\begin{array}{l}\text { Mean } 1.4 \\
\text { (range } 0-4 \text { ) }\end{array}$ & $\begin{array}{l}\text { Mean } 1.6 \\
\text { (range } 1-5 \text { ) }\end{array}$ & $\begin{array}{l}\text { Mean } 1.6 \\
\text { (range } 1-5 \text { ) }\end{array}$ \\
\hline Central (CK7) & $\begin{array}{l}\text { Mean } 7.5 \\
\text { (range } 0-40 \text { ) }\end{array}$ & $\begin{array}{l}\text { Mean } 8.1 \\
\text { (range } 2-7 \text { ) }\end{array}$ & $\begin{array}{l}\text { Mean } 5.1 \\
\text { (range 1-6) }\end{array}$ \\
\hline Peripheral $(\mathrm{H} \& \mathrm{E})$ & $\begin{array}{l}\text { Mean } 0.8 \\
\text { (range } 0-3 \text { ) }\end{array}$ & $\begin{array}{l}\text { Mean } 0.6 \\
\text { (range 0-3) }\end{array}$ & $\begin{array}{l}\text { Mean } 0.5 \\
\text { (range 0-3) }\end{array}$ \\
\hline Peripheral (CK7) & $\begin{array}{l}\text { Mean } 9.0 \\
\text { (range 0-9) }\end{array}$ & $\begin{array}{l}\text { Mean } 11.9 \\
\text { (range } 4-7 \text { ) }\end{array}$ & $\begin{array}{l}\text { Mean } 6.63 \\
\text { (range 2-6) }\end{array}$ \\
\hline
\end{tabular}

${ }^{\star}$ Mean counts in two triads.

$\mathrm{CH}$, chronic hepatitis; PBC, primary biliary cirrhosis.

Table 2 The number of central bile ducts found in two consecutive portal triads in 99 liver biopsies stained with anticytokeratin $7(n=99)$

\begin{tabular}{llll}
\hline No. of central bile ducts & $\begin{array}{l}P B C \\
(n=37)\end{array}$ & $\begin{array}{l}C H \\
(n=43)\end{array}$ & $\begin{array}{l}\text { Miscellaneous } \\
(n=19)\end{array}$ \\
\hline One & 14 & 0 & 8 \\
Two & 28 & 16 & 48 \\
Three & 24 & 42 & 20 \\
$\geqslant$ Four & 210 & 290 & 20 \\
All ducts/case (mean/case) & $276 / 37(7.46)$ & $348 / 43(8.09)$ & $96 / 19(5.05)$ \\
No. cases without central bile ducts & 8 & 0 & 0 \\
\hline
\end{tabular}

$\mathrm{CH}$, chronic hepatitis; PBC, primary biliary cirrhosis.

Table 3 The total number of peripheral bile ducts in two consecutive portal triads, in 99 liver biopsies stained with anticytokeratin 7 (CK7)

\begin{tabular}{llll}
\hline No. of peripheral bile ducts & PBC & CH & Miscellaneous \\
\hline One & 0 & 0 & 0 \\
Two & 4 & 0 & 52 \\
Three & 30 & 0 & 28 \\
Four & 48 & 8 & 24 \\
Five & 70 & 10 & 10 \\
$\geqslant$ Six & 180 & 492 & 12 \\
All peripheral ducts/case (mean) & $332 / 37(8.97)$ & $510 / 43(11.86)$ & $126 / 19(6.63)$ \\
No. cases without peripheral bile ducts & 3 & 0 & 0 \\
\hline
\end{tabular}

$\mathrm{CH}$, chronic hepatitis; PBC, primary biliary cirrhosis.

Table 4 Number of cases having central bile ducts with lumen in two consecutive portal triads. Liver biopsies $(n=99)$ were stained with anticytokeratin 7

\begin{tabular}{lccc}
\hline Cases with central bile ducts in the triads & $\begin{array}{c}P B C \\
(n=37)\end{array}$ & $\begin{array}{l}\text { CH } \\
(n=43)\end{array}$ & $\begin{array}{l}\text { Miscellaneous } \\
(n=19)\end{array}$ \\
\hline With lumen & 5 & 40 & 17 \\
With and without lumen & 16 & 3 & 1 \\
Without lumen & 8 & 0 & 1 \\
All & 29 & 43 & 19 \\
No. cases without central bile ducts & 8 & 0 & 0
\end{tabular}

$\mathrm{CH}$, chronic hepatitis; PBC, primary biliary cirrhosis.

Table 5 Number of cases having peripheral bile ducts with lumen in two consecutive portal triads. Liver biopsies $(n=99)$ were stained with anticytokeratin 7

\begin{tabular}{lccc}
\hline Cases with peripheral bile ducts in the triads & $\begin{array}{l}P B C \\
(n=37)\end{array}$ & $\begin{array}{l}\text { CH } \\
(n=43)\end{array}$ & $\begin{array}{l}\text { Miscellaneous } \\
(n=19)\end{array}$ \\
\hline With lumen & 2 & 0 & 0 \\
With and without lumen & 2 & 2 & 7 \\
Peripheral without lumen & 30 & 41 & 10 \\
All & 34 & 43 & 17 \\
No. cases without peripheral bile ducts & 3 & 0 & 0 \\
\hline
\end{tabular}

$\mathrm{CH}$, chronic hepatitis; PBC, primary biliary cirrhosis.

$(100 \%)$ had $\geqslant 4$ peripheral bile ducts/triad, only two of the 19 miscellaneous cases $(10.5 \%)$ had $\geqslant 4$ peripheral bile ducts/triad.

QUALITATIVE RESULTS

Cases having central bile ducts with lumen (CK7 stained)

Table 4 shows that of the 37 primary biliary cirrhosis cases, five $(13.5 \%)$ had centrally located ducts with lumen, $16(43.2 \%)$ had central ducts with and without lumen, and the remaining eight $(21.6 \%)$ had central ducts without lumen. In the remaining eight cases, central ducts were not found in the triads.

Of the 43 cases with chronic hepatitis, 40 $(93.0 \%)$ had central ducts with lumen and the remaining three $(7.0 \%)$ had some central ducts with and some without lumen (table 4).

Of the 19 miscellaneous cases, 17 (89.5\%) had central bile ducts with lumen, one other case $(5.3 \%)$ had central bile ducts with and others without lumen, and in the remaining case $(5.3 \%)$ all central bile ducts lacked a lumen. Significantly more patients with chronic hepatitis and miscellaneous cases had centrally located ducts with lumen than did patients with primary biliary cirrhosis $(\mathrm{p}<0.001)$.

Cases with peripheral bile ducts with lumen (CK7 stained)

Table 5 shows that of the 37 primary biliary cirrhosis cases, two $(4.6 \%)$ had peripheral ducts with lumen, two $(4.6 \%)$ had peripheral ducts with and without lumen, and $30(81.1 \%)$ had peripheral ducts without lumen. In the remaining three cases, peripheral ducts were not found in the triads. In those three cases, central bile ducts were also missing.

Of the 43 cases with chronic hepatitis, two $(4.6 \%)$ had peripheral ducts with and without lumen and the remaining $41(95.3 \%)$ only had peripheral ducts without lumen. Of the 19 miscellaneous cases, seven (3.6\%) had peripheral bile ducts with and without lumen, and the remaining 12 cases $(63.2 \%)$ had peripheral bile ducts without a lumen. The difference between primary biliary cirrhosis/chronic hepatitis cases having peripheral located ducts with lumen was significantly higher than for miscellaneous cases $(p<0.05)$.

\section{Discussion}

The inflammatory destruction, as well as the absence of bile ducts in the triads, is regarded as an important histological criterium in the diagnosis of primary biliary cirrhosis. ${ }^{12}$ In contrast, the bile ducts are not destroyed by the inflammation in chronic hepatitis. ${ }^{3}$ In the latter disease, it is the surrounding liver parenchyma that is destroyed by the inflammatory process.

Despite these microscopic differences, several authors point out that the differential histological diagnosis between primary biliary cirrhosis and virus or autoimmune induced chronic hepatitis can be very difficult in some cases. $^{1-3}$

We found that in $\mathrm{H} \& \mathrm{E}$ stained sections, many central and peripheral bile ducts in the triads are hidden by the inflammatory cells. This was found not only in primary biliary cirrhosis and chronic hepatitis but also in other liver diseases, and was mainly the result of pronounced lymphocytic crowding, which hampered the visualisation of existing bile ducts in H\&E stained preparations. Those ducts became readily apparent when consecutive sections were stained with anti-CK7. Using this method, the bile ducts were clearly demarcated against the weakly stained background and therefore were easy to identify. 
With the use of anti-CK7, a fivefold increase in the number of central bile ducts (when compared with H\&E stained sections) was recorded for primary biliary cirrhosis and chronic hepatitis and a threefold increase was seen in miscellaneous liver diseases. As regards the number of peripheral bile ducts, a 10 -fold increase in the number was recorded in primary biliary cirrhosis, almost a 20-fold increase in chronic hepatitis, and a 10-fold increase in miscellaneous liver diseases.

In three of the 37 cases $(8.1 \%)$ of primary biliary cirrhosis, bile ducts were not present in the triads, neither in H\&E nor in CK7 stained sections, and in five additional cases (13.5\%) central bile ducts were missing. Conversely, all cases with chronic hepatitis or with miscellaneous liver diseases had central and peripheral bile ducts in the triads.

Of particular interest was the finding that nearly $90 \%$ of the cases with primary biliary cirrhosis and all (100\%) cases with chronic hepatitis had $\geqslant 4$ peripheral bile ducts/triad, whereas only $36.5 \%$ of the miscellaneous cases had a similar number of peripheral bile ducts/triad. It appears that the proliferation of bile ducts at the periphery of the triads might be an important phenomenon in primary biliary cirrhosis and chronic hepatitis.

Not only the quantity but also the quality of the central bile ducts appear to be an important parameter in the differential diagnosis between primary biliary cirrhosis, chronic hepatitis, and other liver diseases. Whereas in primary biliary cirrhosis only $27 \%$ of the cases had central bile ducts with lumen, about $90 \%$ of the cases with chronic hepatitis and with miscellaneous liver diseases had bile ducts with lumen.

Conversely, most of the peripheral bile ducts in primary biliary cirrhosis (89\%) and in chronic hepatitis $(95 \%)$ had no lumen, whereas only $58 \%$ of cases with miscellaneous liver diseases had peripheral bile ducts without lumen. The causes of these differences remain elusive.

In conclusion, anti-CK7 seems to label all bile ducts in the portal triads, independently of the number of chronic inflammatory cells present in that area.

A considerable number of otherwise concealed central and peripheral bile ducts in the triads in $\mathrm{H} \& \mathrm{E}$ stained sections become readily apparent when stained with anti-CK 7 . One possible explanation for this increased detection of peripheral bile ducts might be the proliferation of the "hepatocellular bile duct stem cell" at the limiting plate in response to piecemeal necrosis, or alterations in the matrix as a result of inflammation. In this context, CK7 positive liver cells could be seen abutting portal spaces in some sections having a high number of peripheral bile ducts. Whether anti-CK7 enables the detection of these hepatocellular bile duct stem cells or of "helper" liver cells, participants in the complex process of biliary duct neoformation, remains to be elucidated. The third possibility is that anti-CK7 shows up biliary cell metaplasia of periportal hepatocytes (as suggested by J Lindholm, 1999, personal communication).

It should be stressed that CK7 positive liver cells abutting portal spaces were not found in sections from the same cases stained for CK19, another marker of bile ducts. The expression of the latter cytokeratin, however, is not as distinct as that of CK7.

The absence of CK7 stained central and/or peripheral bile ducts and the lack of lumen in central bile ducts are valuable additional parameters in the differential diagnosis between primary biliary cirrhosis and chronic hepatitis. Anti-CK7 has been incorporated in the past four years into the panel of stains used in the routine histo-immunological diagnosis of liver diseases at this hospital.

This study was supported by the Karolinska Institute and Grant 97:109 from the Cancer Society Stockholm.

1 Kaplan MM. Primary biliary cirrhosis [review]. $N$ Engl $\mathcal{F}$ Med 1996;335:1570-80.

2 Portmann BC, MacSween RNM. Diseases of the intrahepatic bile ducts. In: MacSween RNM, Anthony PP, Scheuer PJ, et al, eds. Pathology of the liver, 3rd ed. Edinburgh: Churchill and Livingstone, 1994:477-512.

3 Rubio CA. The detection of bile ducts in liver biopsies by cytokeratin 7. In Vivo 1998;12:183-6.

4 Rubio CA, Porwit A. Quantification of fibrosis in liver biopsies. Anal Quant Cytol Histol 1988;10:107-9.

5 Nohlgård C, Rubio CA, Kock Y, et al. Liver fibrosis quantified by image analysis in methotrexate-treated patients with psoriasis. F Am Acad Dermatol 1993;28:40-5.

6 Batts KP, Ludwig J. Chronic hepatitis. An update on terminology and reporting. Am $\mathcal{7}$ Surg Pathol 1995;19:1409-17.

7 Jones DEJ, Bassendine MF Antikeratin antibodies (AKA) negativity in primary biliary cirrhosis (PBC): confirmation of their specificity in the diagnosis of rheumatoid arthritis. Clin Rheumatol 1996;15:617-18. 